\title{
Dosimetric Comparison of Radiation Techniques for Comprehensive Regional Nodal Radiation Therapy for Left-Sided Breast Cancer: A Treatment Planning Study
}

Heejoo Ko

Catholic University of Korea College of Medicine: Catholic University of Korea School of Medicine Jee Suk Chang

Yonsei University College of Medicine

Jin Young Moon

Yonsei Cancer Center

Won Hee Lee

Yonsei Cancer Center

Chirag Shah

Taussig Cancer Institute

Jin Sup Andy Shim

New York Proton Center

Min Cheol Han

Yonsei Cancer Center

Jong Geol Baek

Yonsei Cancer Center

Ryeong Hwang Park

Yonsei Cancer Center

\section{Yong Bae Kim}

Yonsei University College of Medicine

Jin Sung Kim ( $\nabla$ JINSUNG@yuhs.ac )

Yonsei University College of Medicine https://orcid.org/0000-0003-1415-6471

\section{Research}

Keywords: Breast cancer, regional nodal irradiation, dosimetric study, volumetric modulated arc therapy, proton beam therapy, continuous positive airway pressure

Posted Date: December 8th, 2020 
DOl: https://doi.org/10.21203/rs.3.rs-120620/v1

License: (c) (1) This work is licensed under a Creative Commons Attribution 4.0 International License. Read Full License 


\section{Abstract}

Background: Radiotherapy (RT) target volumes in breast cancer treatment increasingly include the internal mammary nodes, potentially increasing doses delivered to the heart and lung. The aim of this study was to compare cardiac and pulmonary doses in three-dimensional conformal radiotherapy (3DCRT), volumetric modulated arc therapy (VMAT), and proton beam therapy (PBT) with and without continuous positive airway pressure (CPAP), delivered in conventional fractionation (CF) or in hypofractionated (HF) schedules.

Methods: In a dataset of 15 patients with left-sided breast cancer, partial wide tangential 3DCRT delivered in CF (50/25) and in HF (40/15) schedules as well as PBT delivered in CF and two-partial arc VMAT delivered in a HF schedule, each under CPAP and free breathing (FB) conditions were extensively examined. Target volume coverage and doses to organs-at-risk (OARs) were calculated for each technique. Outcomes were compared with one-way analysis of variance and the Bonferroni test, with Pvalues $<0.05$ considered significant.

Results: Target volume coverage was within acceptable levels in all interventions, except for internal mammary lymph nodes D90 (99\% in PBT, 90\% in VMAT-CPAP, 84\% in VMAT-FB, and 74\% in 3DCRT). Mean heart dose (MHD) was the lowest and highest in PBT ( $<1 \mathrm{~Gy}$ ) and 3DCRT with CF/FB (7.8 Gy), respectively. In photon-based techniques, the use of VMAT in HF schedules with CPAP resulted in an MHD of $2.2 \mathrm{~Gy}$. Lung V20 and mean lung dose (MLD) were the highest in 3DCRT-CF-FB (44\% and $20 \mathrm{~Gy}$, respectively) and lowest in both VMAT-HF-CPAP and PBT (7-9\% and approximately 5-6 Gy, respectively). VMAT-HF-CPAP and PBT delivered a comparable maximum dose to the left ascending artery (7.2 Gy and $6.70 \mathrm{~Gy}$, respectively, with the highest dose delivered by 3DCRT [47.6 Gy]). The average doses to the contralateral breast were $0.004,1$, and $2.1 \mathrm{~Gy}$ in PBT, 3DCRT, and VMAT, respectively.

Conclusions: PBT delivered the lowest doses to most OARs in RT for left-sided breast cancer with regional nodal irradiation. However, the dosimetric gap between proton and photon RT has narrowed substantially with modern techniques such as VMAT and CPAP; the clinical relevance of these differences is yet to be elucidated.

\section{Background}

Modern multi-disciplinary treatment for breast cancer, including radiation therapy (RT), has improved patients' long-term survival rates [1]. Concurrently, delayed side effects associated with RT are gaining more importance. Recent studies have reported that the risk of secondary lung cancer and cardiovascular toxicity is associated with the mean radiation dose delivered to the heart and lung [2]. According to Darby et al., the risk of major coronary events increases linearly by $7.4 \%$ per 1 gray (Gy) increase in the mean heart dose (MHD) [3]. The use of comprehensive regional nodal irradiation (RNI) in RT has increased after evidence from landmark trials became available $[4,5]$. However, extended RT increases the MHD, thus increasing the risk of major coronary events [6]. 
Recently, RT techniques such as deep inspiration breath hold (DIBH), prone RT, cardiac blocking, and continuous positive airway pressure (CPAP) - a new alternative to the $\mathrm{DIBH}$ technique-have been introduced to help reduce the MHD [7, 8]. Fixed-beam intensity-modulated radiotherapy (IMRT), volumetric modulated arc therapy (VMAT), and proton beam therapy (PBT) can also help reduce the MHD. In light of the accumulating evidence regarding the safety and effectiveness of hypofractionated radiotherapy (HFRT), the 3-week schedule of 40 Gy in 15 fractions is being increasingly selected in the treatment of breast cancer, especially during the current coronavirus disease (COVID-19) pandemic [9]. Rotational IMRT such as VMAT has been suggested to reduce the dose to cardiac substructures in the hypofractionated (HF) group [10]. However, how they (cardiac sparing techniques, IMRT/PBT, and HF-RT) affect dose avoidance to the organs-at-risk (OARs) individually or in any combination is poorly understood.

This study aimed to compare target volume coverage and dose to OARs, including cardiac and pulmonary doses, in eight combinations of different RT techniques and dose schedules (threedimensional conformal RT [3DCRT], VMAT, and PBT, with and without CPAP, delivered in a conventional fractionation [CF] or HF schedule) to establish an optimal technique for RNI in the modern era.

\section{Methods}

Fifteen patients with left-sided breast cancer treated with regional nodal RT between April and May 2020 were included. Three patients had undergone mastectomy without reconstruction, while the remaining patients had undergone lumpectomy. All patients underwent computed tomography (CT) with freebreathing (FB) and CPAP. Clinical target volumes of the whole breast axillary lymph nodes (AXL1, AXL2, and AXL3), supraclavicular lymph nodes (SCLs), and internal mammary lymph nodes (IMNs) were delineated separately using CT images. The extent of RNI was modified at the physician's discretion. Planning target volumes (PTVs) were generated with 3-5-mm non-uniform margins based on clinical target volumes maintaining a minimum distance of 3-5 $\mathrm{mm}$ from the skin and lungs. OARs, including the heart, left anterior descending artery (LAD), ipsilateral lung, and contralateral breast, were delineated.

Eight plans were generated for each patient: 3DCRT in CF and HF schedules, VMAT in an HF schedule, and PBT in a CF schedule, using FB- and CPAP-based CT scans. Moreover, 3DCRT in a CF schedule was planned at a dose of $50.0 \mathrm{~Gy}$ in 25 fractions, followed by 5 fractions of sequential boost irradiation at 2.0 Gy per fraction. Meanwhile, 3DCRT in an HF schedule was set to $40.05 \mathrm{~Gy}$ in 15 fractions, followed by 5 fractions of sequential boost irradiation at $2.0 \mathrm{~Gy}$ per fraction. VMAT in an HF schedule was planned as $40.05 \mathrm{~Gy}$ in 15 fractions, with simultaneous integral boost (SIB) to targets at $7.95 \mathrm{~Gy}$ (a total of $48.0 \mathrm{~Gy}$ for boost volume). PBT in CF was planned as 28 fractions of $50.4 \mathrm{~Gy}$ (considering the relative biological effectiveness) with $10 \mathrm{~Gy}$ of SIB added for boost-prescribed patients.

Based on our previous dosimetric study, a partially wide tangential field (PWTF) was selected and generated in the Pinnacle system as the representative of 3DCRT in this study [11]. Medial and lateral 6 MV tangential fields were used to target the breast and IMN. The dose fields were normalized in the same way as the three-field technique photon field. A dose of $6 \mathrm{MV}$ was normalized at a point of the central 
axis of the fields. If any part of the heart was included in the tangential fields, a multileaf collimator was used to shield it from the photon fields. The humeral head, larynx, and trachea were also shielded by the multileaf collimator.

For VMAT, double coplanar arc plans were generated on RayStation (RayStation 5.0.3.17, RaySearch Laboratories AB, Stockholm, Sweden). A linear accelerator with $6 \mathrm{MV}$ photon beams (Versa HD, Elekta, UK) was used in this study. Arc 1 and Arc 2 were simultaneously rotated in the reverse direction. In most cases, Arc 1 started at $295-305^{\circ}$ and stopped at $155-165^{\circ}$. All plans were normalized to ensure that at least $95 \%$ of the chest wall PTV received $95 \%$ of the prescribed dose. The planning criteria for PTV coverage and dose to normal organs were based on institutional practice guidelines. To minimize the occurrence of hotspots, particularly in the CTV, the maximum dose to any point was limited to $105 \%$. RayStation was used for dose calculation and optimization, in a dose grid sized $2 \mathrm{~mm}$ with a collapsed cone algorithm.

Doses to normal tissues were limited as follows: an MHD of < $5 \mathrm{~Gy}$; a maximum and mean dose to the LAD of $<12$ Gy and $<5$ Gy, respectively; $<50 \%,<35 \%$, and $<20 \%$ for the ipsilateral lung volume receiving over 5 Gy (V5Gy), 10 Gy (V10Gy), and 20 Gy (V20Gy), respectively; and finally, a mean contralateral lung dose of $<2-3 \mathrm{~Gy}$. Moreover, wherever possible, doses to the LAD, heart, and other normal tissues were kept as low as possible while ensuring PTV coverage.

PBT plans were generated using the pencil beam scanning technique in Raystation9A (RaySearch Laboratories $\mathrm{AB}$ ). Two beams, including one anterior beam and one left oblique beam at an angle ranging from $30^{\circ}$ to $45^{\circ}$, were used. For PBT plans, the area $5 \mathrm{~mm}$ inside the boundary of the external contour was excluded from PTVs to spare the skin, and a margin of $1 \mathrm{~mm}$ was added with respect to the specific distal and proximal beams. Isotropic 5-mm uncertainty and $3.5 \%$ range uncertainty were accounted for in the robust optimization algorithm. Dose criteria were set based on a combination of the Radiotherapy Comparative Effectiveness Consortium Trial (RADCOMP) description and clinical guidelines of the New York Proton Center. At least $95 \%$ of each PTV was expected to receive $>95 \%$ of the prescribed dose. The maximum dose to the PTV was restricted to $110 \%$ of the prescribed dose. The MHD was set as $<15$ Gy and ipsilateral lung V20Gy was set as $<50 \%$ to comply with the mandatory constraints criteria of RADCOMP [12]. However, wherever possible, the ipsilateral lung V20Gy of $<15 \%$, MHD of $<2 \mathrm{~Gy}$, maximum dose to the heart surface of $<20 \mathrm{~Gy}$, and maximum dose to the skin of $<95 \%$ of the prescribed dose were used as planning objectives.

Target volume coverage and doses to OARs were calculated for each RT technique. Target volume coverage was evaluated with D90 (dose to $90 \%$ of the target volume), D95 (dose to $95 \%$ of the target volume), homogeneity index ( $\mathrm{HI})$, and conformity index (Cl). D90 and D95 were reported as the percentage of the prescribed dose, as the prescribed doses differed between techniques. Composite plans with individual PTVs for the whole breast, boost, AXL nodes, SCLs, and IMNs were evaluated separately for each target volume. Doses to the heart, LAD, ipsilateral lung, contralateral breast, and skin were calculated for dose evaluation for OARs. The 5-mm zone inside the boundary of the external contour was 
considered the skin area. All 120 plans met the mandatory constraint criteria for PTVs and OARs, as described. The resulting parameter estimates were compared using one-way analysis of variance and the Bonferroni test using R (v.4.0.2, The R Foundation for Statistical Computing, Vienna, Austria) in RStudio (v.1.3, RStudio Inc., Boston, MA). P-values of $<0.05$ were considered indicative of statistical significance.

\section{Results}

The representative cross-sectional dose distributions of all eight techniques in a single patient on the same level cut of CT images are illustrated in Fig. 1. Figure 2 depicts the mean dose-volume histograms of 15 patients for each of the eight techniques with respect to key PTVs and OARs. The mean values of PTV coverage and OARs of interest in 15 patients are presented in Supplementary Table 1.

Regarding PTV coverage, PBT and VMAT were superior in terms of D90, D95, $\mathrm{HI}$, and $\mathrm{Cl}$ to 3DCRT, with either an HF or CF schedule. There was no notable difference in boost coverage between any techniques. In contrast to coverage for the other nodes, which followed the tendency observed for whole PTV coverage, PBT achieved greater coverage of IMNs than VMAT, with statistical significance (in D90 and D95). No significant difference between the use of CPAP and FB was observed in any PTV coverage results (Fig. $3 g$ and h).

The MHD values varied considerably between techniques. PBT with CPAP achieved the lowest MHD (0.34 Gy). Compared to the use of FB, the use of CPAP reduced MHD in every technique, except for PBT. In VMAT with CPAP, the mean MHD was $2.21 \mathrm{~Gy}$, which was $44 \%$ lower than the corresponding value achieved with VMAT in FB conditions (3.94 Gy) and which was non-inferior to that achieved with PBT in FB conditions (Fig. 3a).

Five techniques including all four 3DCRT techniques and VMAT in FB conditions showed maximum LAD doses of $>20 \mathrm{~Gy}$, while the other three techniques achieved doses of $<10 \mathrm{~Gy}$. Among photon-based techniques, only VMAT with CPAP achieved the maximum LAD dose that was non-inferior to that achieved by PBT (Fig. 3b).

VMAT and PBT techniques significantly reduced the mean ipsilateral lung dose (MLD) compared with that achieved with 3DCRT. Regarding MLD, no significant difference was found with the use of CPAP, in comparison to the use of relevant RT techniques with FB. PBT in FB conditions attained the lowest MLD (4.48 Gy), while VMAT-based techniques achieved an MLD of 6.67 Gy (Fig. 3c).

The percentage of the ipsilateral lung volume receiving over 20 Gy (V20) was lower in VMAT and PBT than in 3DCRT. The corresponding values were comparable between VMAT and PBT techniques (approximately 7-9\%) (Fig. 3d). Figure 4 presents the estimates of the lowest ipsilateral lung V5 (volume receiving $>5$ Gy) achieved with PBT techniques.

In PBT techniques, $103-105 \%$ of the prescribed dose was administered to $1 \%$ of the volume of the skin (D1\%); this technique was superior to all 3DCRT techniques but comparable to VMAT techniques 
(Fig. 3e).

VMAT techniques achieved a mean contralateral breast dose of $>2.1 \mathrm{~Gy}$, which was greater than those achieved by the other techniques. In contrast, PBT techniques achieved a mean contralateral breast dose of $<0.005 \mathrm{~Gy}$, which was lower than those achieved by the other techniques (Fig. 3f). Findings for the OARs are summarized in Fig. 5.

\section{Discussion}

Disease-free survival in patients with breast cancer has improved by $3-5 \%$, according to the results of modern clinical trials involving RNI, including RNI of SCLs, AXLs, and IMNs [4, 5]. Maximizing the therapeutic efficacy of RNI requires the improvement of the accuracy of dose delivery to the target volumes, while sparing the surrounding OARs, which may be achieved with modern techniques used individually or in combination. In this study, we compared three RT techniques (standard 3D photon, VMAT, and PBT) with or without CPAP delivered in CF or HF schedules to 15 patients with left-sided breast cancer whose regional nodes were being treated.

In the present study, 3DCRT (PWTF in this study) in FB conditions, as the representative of conventional treatment, showed the following dosimetry profile: the D95 of the PTV was $81 \%$ of the prescribed dose, with a $\mathrm{Cl}$ of 0.52 , skin D1\% of $113 \%$, maximum LAD dose of $48 \mathrm{~Gy}$, and MLD of $20 \mathrm{~Gy}$. The MHD of 7.8 Gy was slightly lower than that previously reported yet consistent with that presented in studies using wide tangents or matched photon-electron fields in FB conditions (approximately $9 \mathrm{~Gy}$ ) [11, 13]. Meanwhile, the discrepancy in findings may be explained by the reduced coverage of IMN target volumes due to partial heart block with a PWTF in the current study (D95 75\%, D90 80\%).

In the present study, PBT was associated with the lowest doses to most OARs and with the best target volume coverage: the D95 of the PTV was $98 \%$ of the prescribed dose, with a $\mathrm{Cl}$ of 0.93 , IMN coverage (D95) of $99 \%$, skin D1\% of $105 \%$, maximum LAD dose of $8.3 \mathrm{~Gy}$, MLD of $4.5 \mathrm{~Gy}$, and MHD of $0.6 \mathrm{~Gy}$, which were consistent with or slightly better than the values previously reported for PBT $[14,15]$. This might be due to the use of two beams, the skin being clipped out of the PTV, or active OAR sparing during RT planning. Nevertheless, the clinical benefit of this excellent dose distribution remains unclear and subject to scrutiny in multi-center randomized trials based in North America (RADCOMP; NCT02603341) and Denmark (The DBCG Proton; NCT04291378).

Standard 3DCRT under FB conditions and PBT are at the opposite ends of the spectrum in terms of MHD. However, it is important to assess the impact of modern photon RT techniques and their relative contributions to any increase in the risk of long-term side effects, as proton beams are not universally accessible or cost-effective in the treatment of women without comorbidities or of those who receive an MHD of $<5$ Gy [16]. The DBCG proton trial is recruiting patients with indications for RT, wherein standard RT planning reveals an MHD of $>4 \mathrm{~Gy}$. 
The dosimetric profile of VMAT with CPAP in an HF schedule reported in the present study is encouraging: the D95 of the PTV was $96 \%$ of the prescribed dose, with a Cl of 0.91 , IMN coverage (D95) of $90 \%$, skin D1\% of $107 \%$, maximum LAD dose of $7.2 \mathrm{~Gy}$, left lung V20 of 9\%, and MHD of $2.2 \mathrm{~Gy}$. In the FB condition, a $50 \%$ reduction in MHD from $7.8 \mathrm{~Gy}$ (3DCRT) to $3.9 \mathrm{~Gy}$ was achieved with the use of VMAT. These values are similar to those previously reported for VMAT with the DIBH technique in a study in the United Kingdom (PTV nodes 96\% and MHD $2.6 \mathrm{~Gy}$ ), with the exception of the maximum LAD dose (23.3 Gy) and left lung dose (V17Gy 28\%) [15].

IMRT has been proposed as a heart sparing technique, although findings regarding MHD have been conflicting. In a recent prospective study from the Memorial Sloan-Kettering Cancer Center [17], an MHD of 13.2 Gy (range, 8.6-20 Gy) was reported in patients with left-sided breast cancer who received multibeam IMRT. In a Korean dummy-run study (KROG 1901), 21 institutions received a representative case and were requested to create a complete RT plan from target delineation according to respective institutional protocols [18]. Although all submitted plans were generated for IMRT (13 fixed-field IMRT, 7 VMAT, and 1 helical tomotherapy), the median MHD was $12.5 \mathrm{~Gy}$ with a wide range (3.3-24.1 Gy). In a study by Pham et al., an MHD of 5.7 Gy was reported with VMAT-DIBH [19].

IMRT techniques vary in sophistication, and discrepancies in MHD can be accounted for by several factors. First, the differences in methods used for the balancing of target volume coverage versus the minimization of MHD in the inverse planning process; second, the use of the DIBH technique or CPAP; third, the use of different fractionation schedules; and fourth, the inter-physician/institution variation in target delineation and PTV setup margins. Quality assurance schemes for RT plans and regular audits with peer review might help establish best practice guidelines for the use of IMRT in breast cancer RT.

Evidence suggests that MHD can be reduced by $50 \%$ with the use of the DIBH technique [20,21]. DIBH is associated with some practical challenges (such as prolonged daily treatment time and the requirement of a patient's cooperation with high compliance). Therefore, we have used CPAP as an alternative to DIBH since 2020 [22]. In the present study, we observed a 53\% reduction in the MHD due to the use of CPAP (MHD of 3DCRT with CPAP compared with those in 3DCRT under FB conditions in either CF or HF schedules); this finding was consistent with those of previous studies. In the present study, the use of VMAT with CPAP achieved an additional $40.1 \%$ reduction in the MHD (compared with those with 3DCRT with CPAP in CF, from $3.7 \mathrm{~Gy}$ to $2.2 \mathrm{~Gy}$ ). Compared with PBT, only VMAT with CPAP showed no significant difference in MHD, which indicates that the gap between photon and proton RT has narrowed substantially with modern RT techniques.

During RT simulation, pressure of CPAP was gradually increased to the highest level tolerated by the patient. An MHD of $1.77 \mathrm{~Gy}$ was observed in VMAT with CPAP at a pressure of $17 \mathrm{~cm} \mathrm{H}_{2} \mathrm{O}$ (versus $2.6 \mathrm{~Gy}$ and $2.3 \mathrm{~Gy}$ achieved at pressures of $15 \mathrm{~cm} \mathrm{H}_{2} \mathrm{O}$ and $12 \mathrm{~cm} \mathrm{H}_{2} \mathrm{O}$, respectively). Furthermore, ipsilateral lung V20 was reduced to $9 \%$ in VMAT with CPAP, a result comparable with that of lung-sparing PBT. 
HF schedules have become a new standard in breast cancer RT, particularly in patients with early-stage disease. Although evidence from a recent randomized trial based in China supported the use of HF schedules in the treatment of locally advanced breast cancer [23], concerns exist over the safety and efficacy profile of HF in RNI settings [24]. For example, the 40/15 regimen, used in the present study, is a popular regimen in RNI settings in the United Kingdom and Korea and was endorsed by the guidelines issued during the COVID-19 pandemic [9]; moreover, it is currently being tested in clinical trials based in Denmark and France (NCT02384733 and NCT03127995). Concurrently, in North America, an HF regimen of $>40 / 15$ in 2 Gy-equivalence is being tested in randomized trials (Alliance A221505 [NCT03414970] and FABREC [NCT03422003]).

In the present study, as a consequence of using the $40 / 15$ regimen instead of the $50 / 25$ regimen, albeit not statistically significant, the MHD decreased by $1.5 \mathrm{~Gy}$ and $0.71 \mathrm{~Gy}$ under FB and CPAP conditions, respectively. The maximum $L A D$ dose showed a significant reduction of $9.3 \mathrm{~Gy}$ and $8.4 \mathrm{~Gy}$ with an HF schedule in FB and CPAP conditions, respectively; this finding is similar to that reported by Pierre et al. [10]. In addition, the $40 / 15 \mathrm{HF}$ schedule can help reduce the lung dose to a greater extent than the 50/25 schedule, a result consistent with that of a previous study [25]. However, the clinical relevance of these dosimetric differences associated with HF regimens is yet to be elucidated.

Regarding the contralateral breast dose, the present findings are consistent with those of previous studies [15]. Only PBT techniques were able to spare the contralateral breast tissue, delivering a dose close to 0 Gy (0.004-0.005 Gy). Although VMAT significantly increased the dose delivered to the contralateral breast compared with that delivered by 3DCRT, the mean dose difference between these techniques was approximately $1 \mathrm{~Gy}$. According to a recent study based on the National Cancer Database, which includes data regarding breast cancer and other tumor types, the relative risk of secondary cancer associated with IMRT is similar to that associated with 3DCRT [26], suggesting that these dose differences may not translate to clinically relevant outcomes in most middle-age and older women.

Overall, these findings suggest that each of the eight techniques can achieve adequate target volume coverage with varying doses delivered to OARs, indicating that RT for breast cancer should be personalized based on each patient's anatomical characteristics. Bazan et al. recently reported the potential of an adaptive treatment planning algorithm for IMRT versus 3DCRT in RNI settings [27]. In the algorithm, IMRT was used in 30\% of patients for whom 3DCRT did not meet the critical OAR constraint criteria. Moreover, Hytonen et al. recently presented the feasibility of an automated patient-specific evidence-based decision-making system for optimizing proton or photon treatment based on normal tissue complication probability [18]. In a simulation of this study, PBT was indicated for $22 \%$ of the patients, and multiple patients were close to the decision threshold at the same time. Taken together, the present findings may contribute to the development of treatment strategies for patients with left-sided breast cancer undergoing RNI.

\section{Conclusion}


In conclusion, among available techniques, modern PBT can achieve the lowest doses to most OARs in the regional nodal treatment of left-sided breast cancer. The gap in the MHD between proton and photon RT has narrowed substantially with modern techniques. In VMAT-HF with CPAP, an MHD and maximum LAD dose of approximately $2 \mathrm{~Gy}$ and $7 \mathrm{~Gy}$, respectively, were achieved with adequate target volume coverage and a reduced lung dose (V20 9\%, MLD 6 Gy). The clinical relevance of these dosimetric differences is yet to be elucidated.

\section{Abbreviations}

3DCRT, three-dimensional conformal radiotherapy

$\mathrm{CF}$, conventional fractionation

CPAP, continuous positive airway pressure

$\mathrm{DIBH}$, deep inspiration breath hold

$F B$, free-breathing

HF, hypofractionated

IMN, internal mammary lymph nodes

IMRT, intensity-modulated radiotherapy

$L A D$, left anterior descending artery

MHD, mean heart dose

MLD, mean lung dose

OAR, organ-at-risk

PBT, proton beam therapy

PWTF, partially wide tangential field

RADCOMP, radiotherapy comparative effectiveness consortium trial

RNI, regional nodal irradiation

$\mathrm{RT}$, radiotherapy

SCL, supraclavicular lymph nodes

VMAT, volumetric modulated arc therapy 


\section{Declarations}

\section{Ethics approval and consent to participate}

This study was approved by the ethics review board of our institution; the requirement to obtain informed consent was waived because the data were anonymized.

\section{Consent for publication}

Not applicable.

\section{Availability of data and materials}

Not applicable

\section{Competing interests}

JSK is a cofounder of Oncosoft and serves as an advisor to Rayence. JSC acts as scientific consultant to Accuray.

\section{Funding}

This work was supported by the National Research Foundation of Korea (NRF) grant funded by the Korea government (MSIT) (No. 2020R1A4A1016619).

\section{Authors' contributions}

HK, JSC and JSK conceived the study and performed all the analysis and drafted the manuscript. JYM, WHL, CS, JSS, MCH, JGB, RHP and YBK critically reviewed the text and contributed to the clinical analysis. All authors approved the final version of the manuscript.

\section{Acknowledgments}

Presented at 2020 annual meeting of Korean Society for Radiation Oncology (KOSRO), October 2020, Seoul, Korea (Online).

\section{References}


1. Bray F, Ferlay J, Soerjomataram I, Siegel RL, Torre LA, Jemal A. Global cancer statistics 2018 : GLOBOCAN estimates of incidence and mortality worldwide for 36 cancers in 185 countries. CA Cancer J Clin. 2018;68(6):394-424.

2. Taylor C, Correa C, Duane FK, Aznar MC, Anderson SJ, Bergh J, et al. Estimating the Risks of Breast Cancer Radiotherapy: Evidence From Modern Radiation Doses to the Lungs and Heart and From Previous Randomized Trials. J Clin Oncol. 2017;35(15):1641-9.

3. Darby SC, Ewertz M, McGale P, Bennet AM, Blom-Goldman U, Bronnum D, et al. Risk of ischemic heart disease in women after radiotherapy for breast cancer. N Engl J Med. 2013;368(11):987-98.

4. Poortmans PM, Collette S, Kirkove C, Van Limbergen E, Budach V, Struikmans H, et al. Internal Mammary and Medial Supraclavicular Irradiation in Breast Cancer. N Engl J Med. 2015;373(4):31727.

5. Whelan TJ, Olivotto IA, Parulekar WR, Ackerman I, Chua BH, Nabid A, et al. Regional Nodal Irradiation in Early-Stage Breast Cancer. N Engl J Med. 2015;373(4):307-16.

6. Taylor CW, Kirby AM. Cardiac Side-effects From Breast Cancer Radiotherapy. Clin Oncol (R Coll Radiol). 2015;27(11):621-9.

7. Shah C, Badiyan S, Berry S, Khan AJ, Goyal S, Schulte K, et al. Cardiac dose sparing and avoidance techniques in breast cancer radiotherapy. Radiother Oncol. 2014;112(1):9-16.

8. Allen AM, Ceder YK, Shochat T, Fenig E, Popovtzer A, Bragilofsky D, et al. CPAP (Continuous Positive Airway Pressure) is an effective and stable solution for heart sparing radiotherapy of left sided breast cancer. Radiat Oncol. 2020;15(1):59.

9. Coles CE, Aristei C, Bliss J, Boersma L, Brunt AM, Chatterjee S, et al. International Guidelines on Radiation Therapy for Breast Cancer During the COVID-19 Pandemic. Clin Oncol (R Coll Radiol). 2020;32(5):279-81.

10. Loap P, Fourquet A, Kirova Y. The Limits of the Linear Quadratic (LQ) Model for Late Cardiotoxicity Prediction: Example of Hypofractionated Rotational Intensity Modulated Radiation Therapy (IMRT) for Breast Cancer. Int J Radiat Oncol Biol Phys. 2020;106(5):1106-8.

11. Jeong K, Shim SJ, You SH, Kim YB, Keum KC, Kim JD, et al. A Study of the Radiotherapy Techniques for the Breast Including Internal Mammary Lymph Nodes. Radiat Oncol J. 2009;27(1):35-41.

12. Bekelman JE, Lu H, Pugh S, Baker K, Berg CD, de Gonzalez AB, et al. Pragmatic randomised clinical trial of proton versus photon therapy for patients with non-metastatic breast cancer: the Radiotherapy Comparative Effectiveness (RadComp) Consortium trial protocol. BMJ Open. 2019;9(10):e025556.

13. Taylor C, McGale P, Bronnum D, Correa C, Cutter D, Duane FK, et al. Cardiac Structure Injury After Radiotherapy for Breast Cancer: Cross-Sectional Study With Individual Patient Data. J Clin Oncol. 2018;36(22):2288-96.

14. Verma V, Iftekaruddin Z, Badar N, Hartsell W, Han-Chih Chang J, Gondi V, et al. Proton beam radiotherapy as part of comprehensive regional nodal irradiation for locally advanced breast cancer. Radiother Oncol. 2017;123(2):294-8. 
15. Ranger A, Dunlop A, Hutchinson K, Convery H, Maclennan MK, Chantler H, et al. A Dosimetric Comparison of Breast Radiotherapy Techniques to Treat Locoregional Lymph Nodes Including the Internal Mammary Chain. Clin Oncol (R Coll Radiol). 2018;30(6):346-53.

16. Mailhot Vega RB, Ishaq O, Raldow A, Perez CA, Jimenez R, Scherrer-Crosbie M, et al. Establishing Cost-Effective Allocation of Proton Therapy for Breast Irradiation. Int J Radiat Oncol Biol Phys. 2016;95(1):11-8.

17. Ho AY, Ballangrud A, Li G, Gupta GP, McCormick B, Gewanter R, et al. Long-Term Pulmonary Outcomes of a Feasibility Study of Inverse-Planned, Multibeam Intensity Modulated Radiation Therapy in Node-Positive Breast Cancer Patients Receiving Regional Nodal Irradiation. Int J Radiat Oncol Biol Phys. 2019;103(5):1100-8.

18. Hytonen R, Koponen TK, Vanderstraeten R, Smith C, Verbakel WFAR, Vergeer MR. Automated patient specific knowledge-based decision making for proton or photon treatment based on NTCP. In. Particle Therapy Co-Operative Group (PTCOG) 2020. Online2020.; 2020.

19. Pham TT, Ward R, Latty D, Owen C, Gebski V, Chojnowski J, et al. Left-sided breast cancer locoregional radiotherapy with deep inspiration breath-hold: Does volumetric-modulated arc radiotherapy reduce heart dose further compared with tangential intensity-modulated radiotherapy? J Med Imaging Radiat Oncol. 2016;60(4):545-53.

20. Bruzzaniti V, Abate A, Pinnaro P, D'Andrea M, Infusino E, Landoni V, et al. Dosimetric and clinical advantages of deep inspiration breath-hold (DIBH) during radiotherapy of breast cancer. J Exp Clin Cancer Res. 2013;32:88.

21. Lee HY, Chang JS, Lee IJ, Park K, Kim YB, Suh CO, et al. The deep inspiration breath hold technique using Abches reduces cardiac dose in patients undergoing left-sided breast irradiation. Radiat Oncol J. 2013;31(4):239-46.

22. Goldstein JD, Lawrence YR, Appel S, Landau E, Ben-David MA, Rabin T, et al. Continuous Positive Airway Pressure for Motion Management in Stereotactic Body Radiation Therapy to the Lung: A Controlled Pilot Study. Int J Radiat Oncol Biol Phys. 2015;93(2):391-9.

23. Wang SL, Fang H, Song YW, Wang WH, Hu C, Liu YP, et al. Hypofractionated versus conventional fractionated postmastectomy radiotherapy for patients with high-risk breast cancer: a randomised, non-inferiority, open-label, phase 3 trial. Lancet Oncol. 2019;20(3):352-60.

24. Marta GN, Coles C, Kaidar-Person O, Meattini I, Hijal T, Zissiadis Y, et al. The use of moderately hypofractionated post-operative radiation therapy for breast cancer in clinical practice: A critical review. Critical Reviews in Oncology/Hematology. 2020;156:103090.

25. Lee BM, Chang JS, Kim SY, Keum KC, Suh CO, Kim YB. Hypofractionated Radiotherapy Dose Scheme and Application of New Techniques Are Associated to a Lower Incidence of Radiation Pneumonitis in Breast Cancer Patients. Front Oncol. 2020;10:124.

26. Xiang M, Chang DT, Pollom EL. Second cancer risk after primary cancer treatment with threedimensional conformal, intensity-modulated, or proton beam radiation therapy. Cancer. 2020;126(15):3560-8. 
27. Bazan JG, Healy E, Beyer S, Kuhn K, DiCostanzo D, Smith TL, et al. Clinical Effectiveness of an Adaptive Treatment Planning Algorithm for Intensity Modulated Radiation Therapy Versus 3D Conformal Radiation Therapy for Node-Positive Breast Cancer Patients Undergoing Regional Nodal Irradiation/Postmastectomy Radiation Therapy. Int J Radiat Oncol Biol Phys. 2020. https://doi.org/10.1016/j.jijrobp.2020.07.027.

\section{Figures}

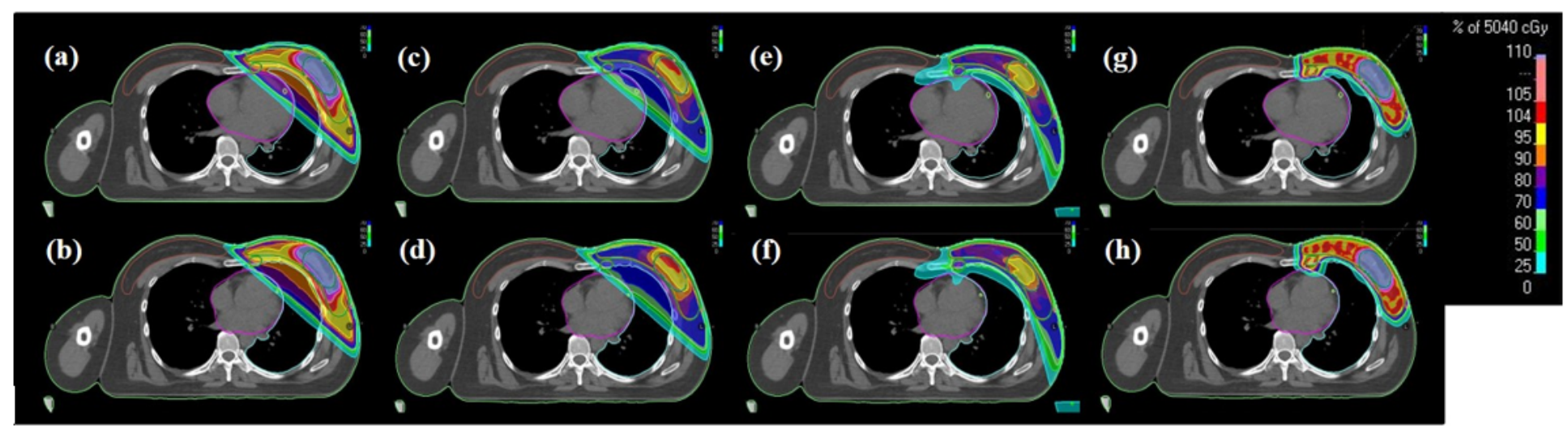

Figure 1

Representative 2D dose distributions in a patient (a) 3DCRT-FB-CF, (b) 3DCRT-CPAP-CF, (c) 3DCRT-FB-HF, (d) 3DCRT-CPAP-HF, (e) VMAT-FB-HF, (f) VMAT-CPAP-HF, (g) PBT-FB-CF, and (h) PBT-CPAP-CF on the same level cut of $C T$ images. 3DCRT, three-dimensional conformal radiotherapy; VMAT, volumetric modulated arc therapy; $\mathrm{PBT}$, proton beam therapy; $\mathrm{CPAP}$, continuous positive airway pressure; $\mathrm{CF}$, conventional fractionation; HF, hypofractionation

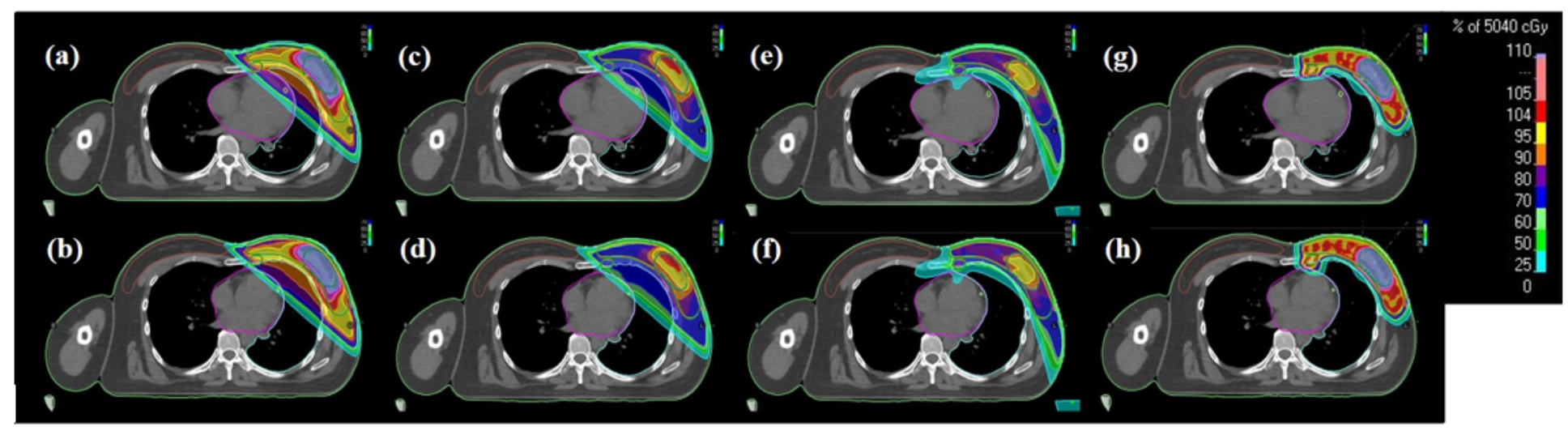

\section{Figure 1}

Representative 2D dose distributions in a patient (a) 3DCRT-FB-CF, (b) 3DCRT-CPAP-CF, (c) 3DCRT-FB-HF, (d) 3DCRT-CPAP-HF, (e) VMAT-FB-HF, (f) VMAT-CPAP-HF, (g) PBT-FB-CF, and (h) PBT-CPAP-CF on the same level cut of CT images. 3DCRT, three-dimensional conformal radiotherapy; VMAT, volumetric modulated 
arc therapy; PBT, proton beam therapy; CPAP, continuous positive airway pressure; CF, conventional fractionation; $\mathrm{HF}$, hypofractionation
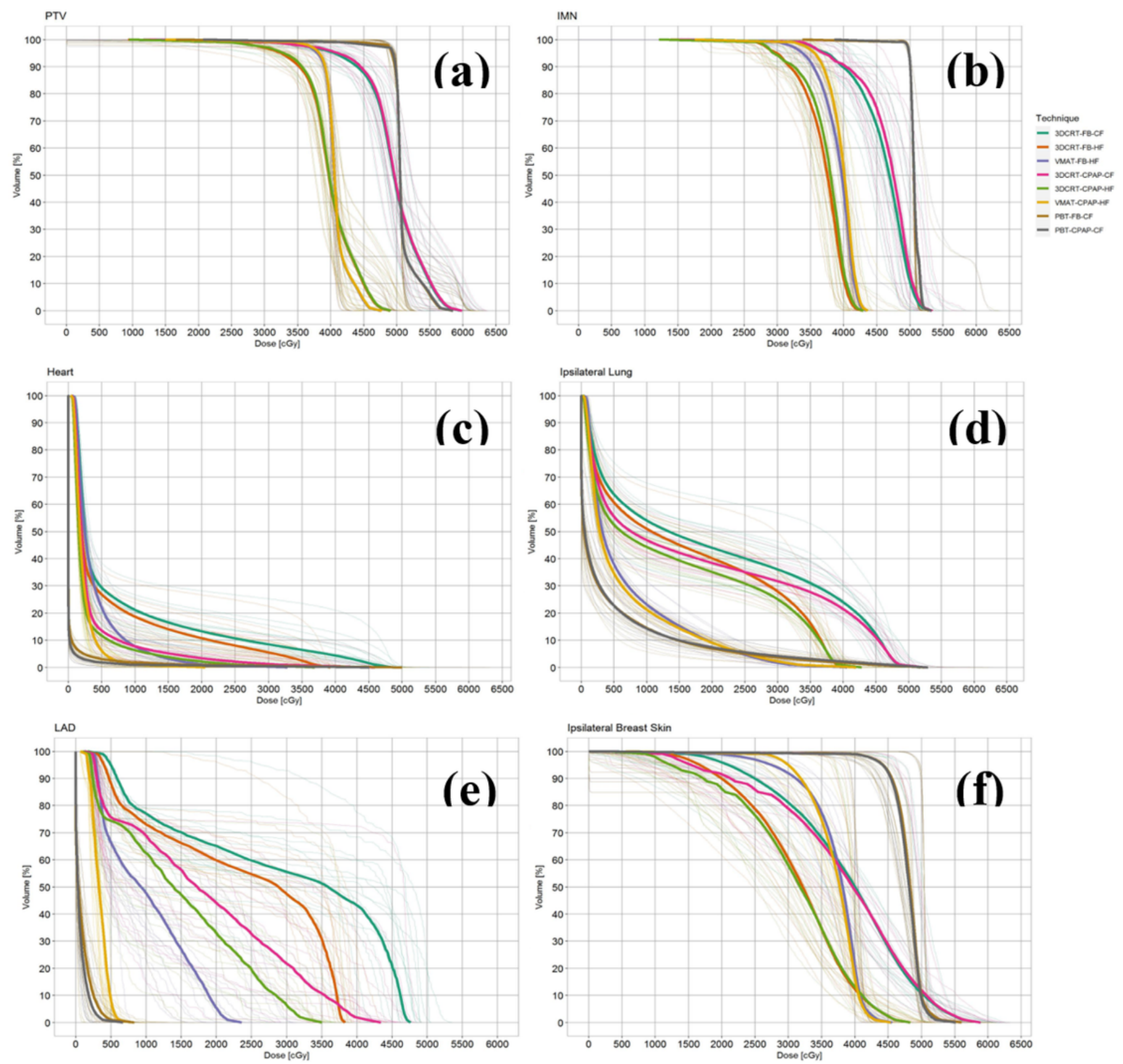

\section{Figure 2}

Dose-volume histograms (DVH) (a) PTV, (b) IMN, (c) heart, (d) ipsilateral lung, (e) LAD, and (f) ipsilateral breast skin. Pale lines represent the DVH per patient per technique; thick lines represent the mean DVH curve of each technique IMN, internal mammary lymph nodes; LAD, left anterior descending artery; PTV, planning target volume 

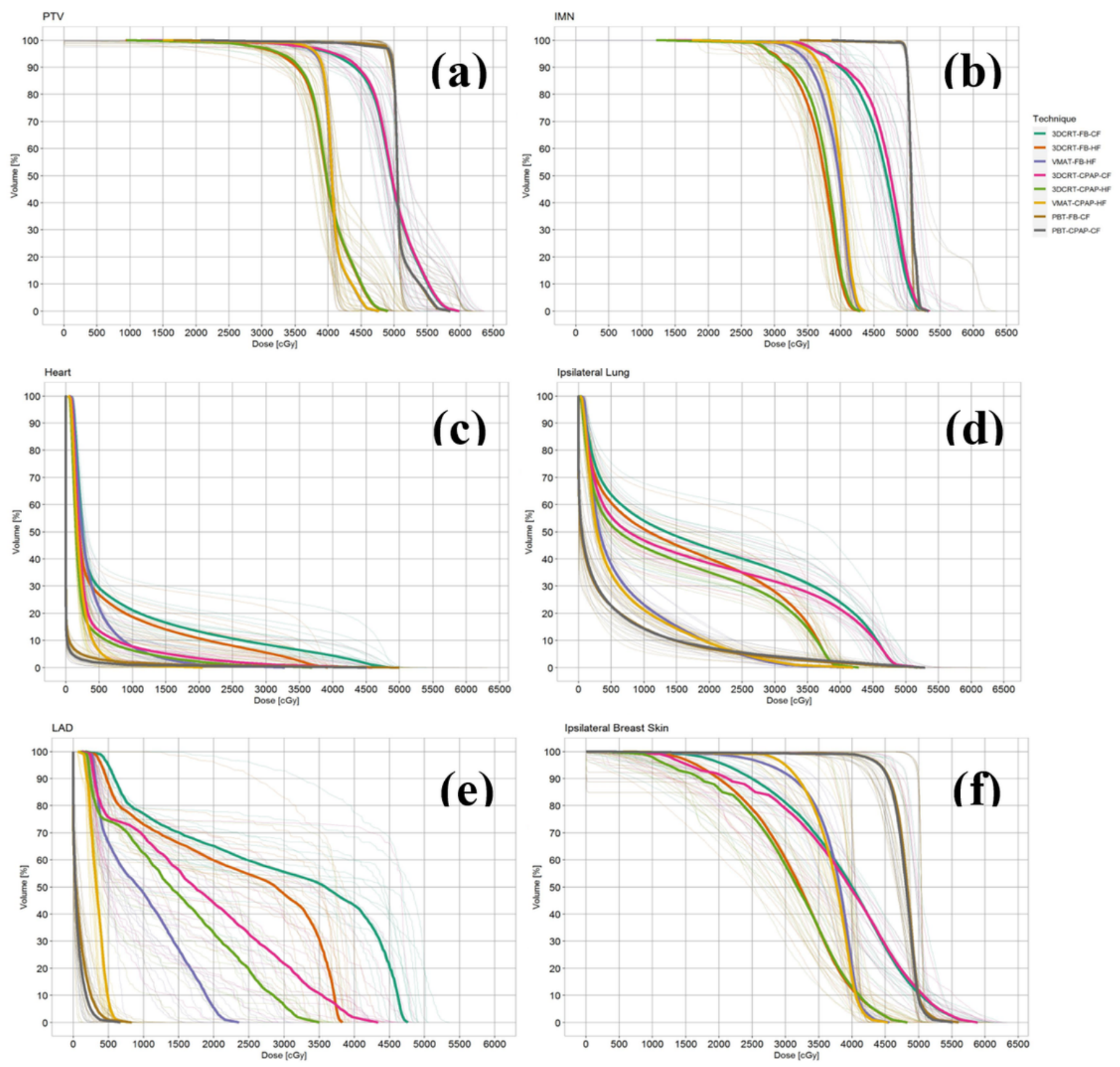

Figure 2

Dose-volume histograms (DVH) (a) PTV, (b) IMN, (c) heart, (d) ipsilateral lung, (e) LAD, and (f) ipsilateral breast skin. Pale lines represent the DVH per patient per technique; thick lines represent the mean DVH curve of each technique IMN, internal mammary lymph nodes; LAD, left anterior descending artery; PTV, planning target volume 


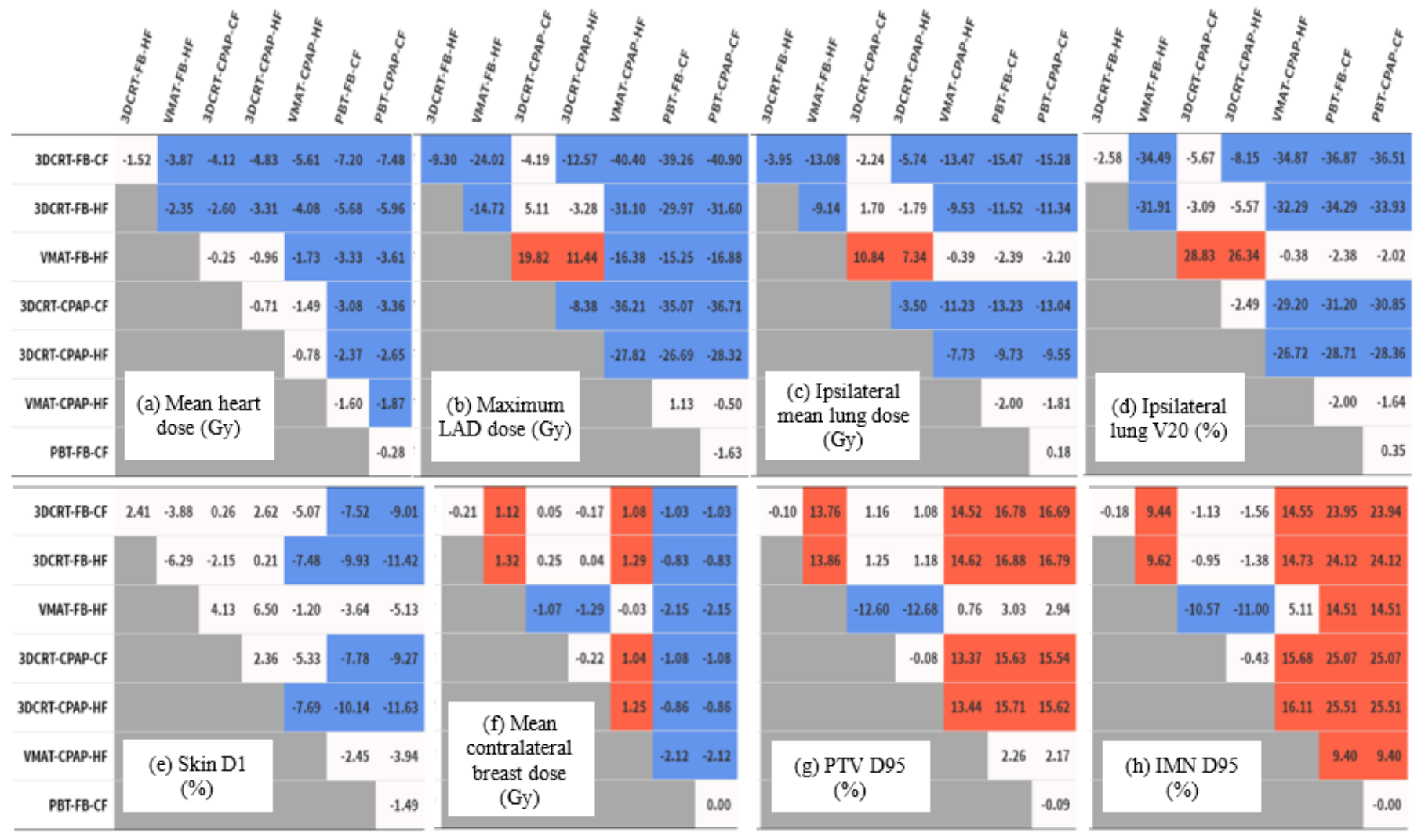

\section{Figure 3}

Differences in dose distribution values The values represent the differences between the values obtained by the techniques listed in the columns and those obtained by the techniques listed in the rows. Red indicates statistically significant-positive differences and blue indicates statistically significant-negative differences 


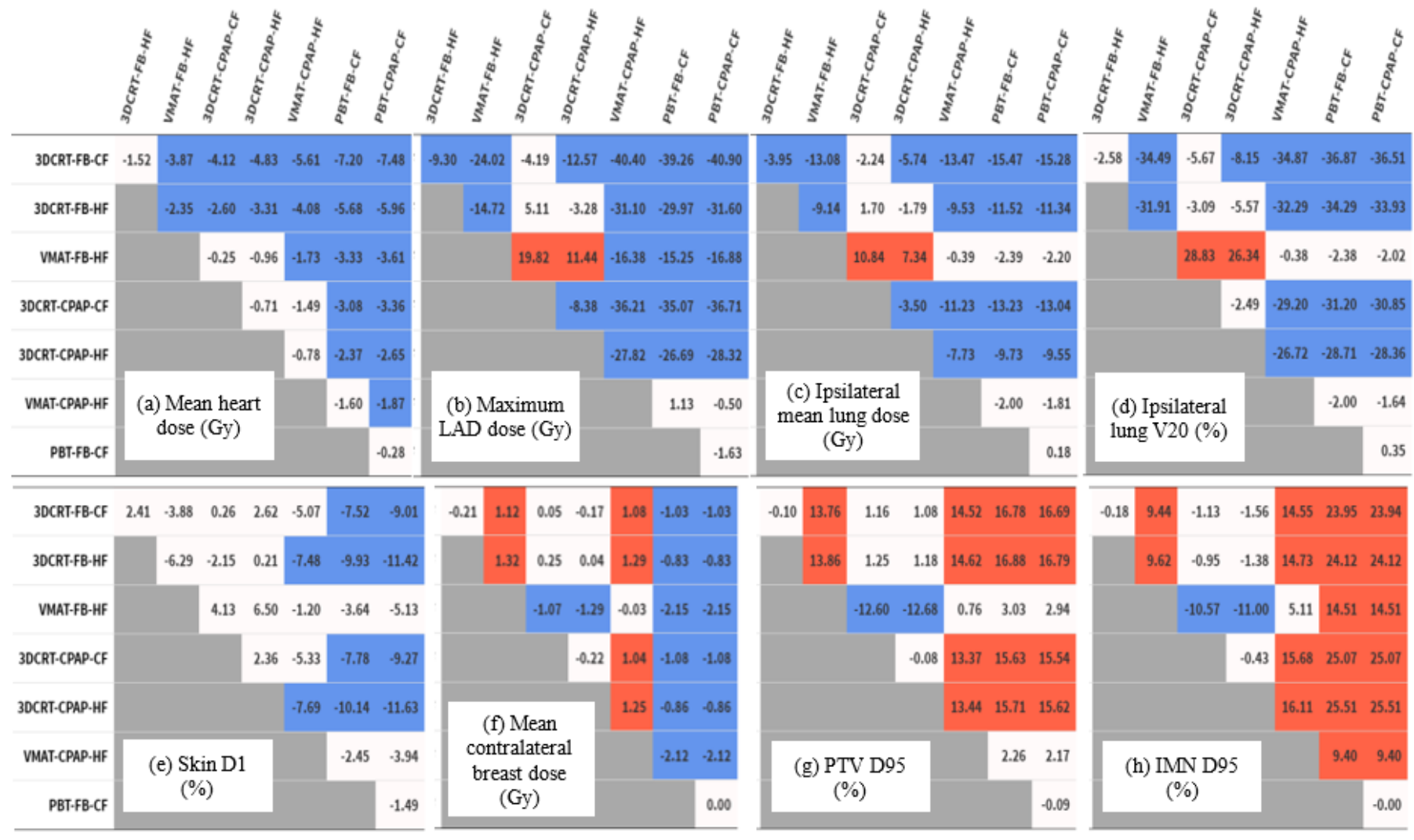

\section{Figure 3}

Differences in dose distribution values The values represent the differences between the values obtained by the techniques listed in the columns and those obtained by the techniques listed in the rows. Red indicates statistically significant-positive differences and blue indicates statistically significant-negative differences 
庰 V5Gy

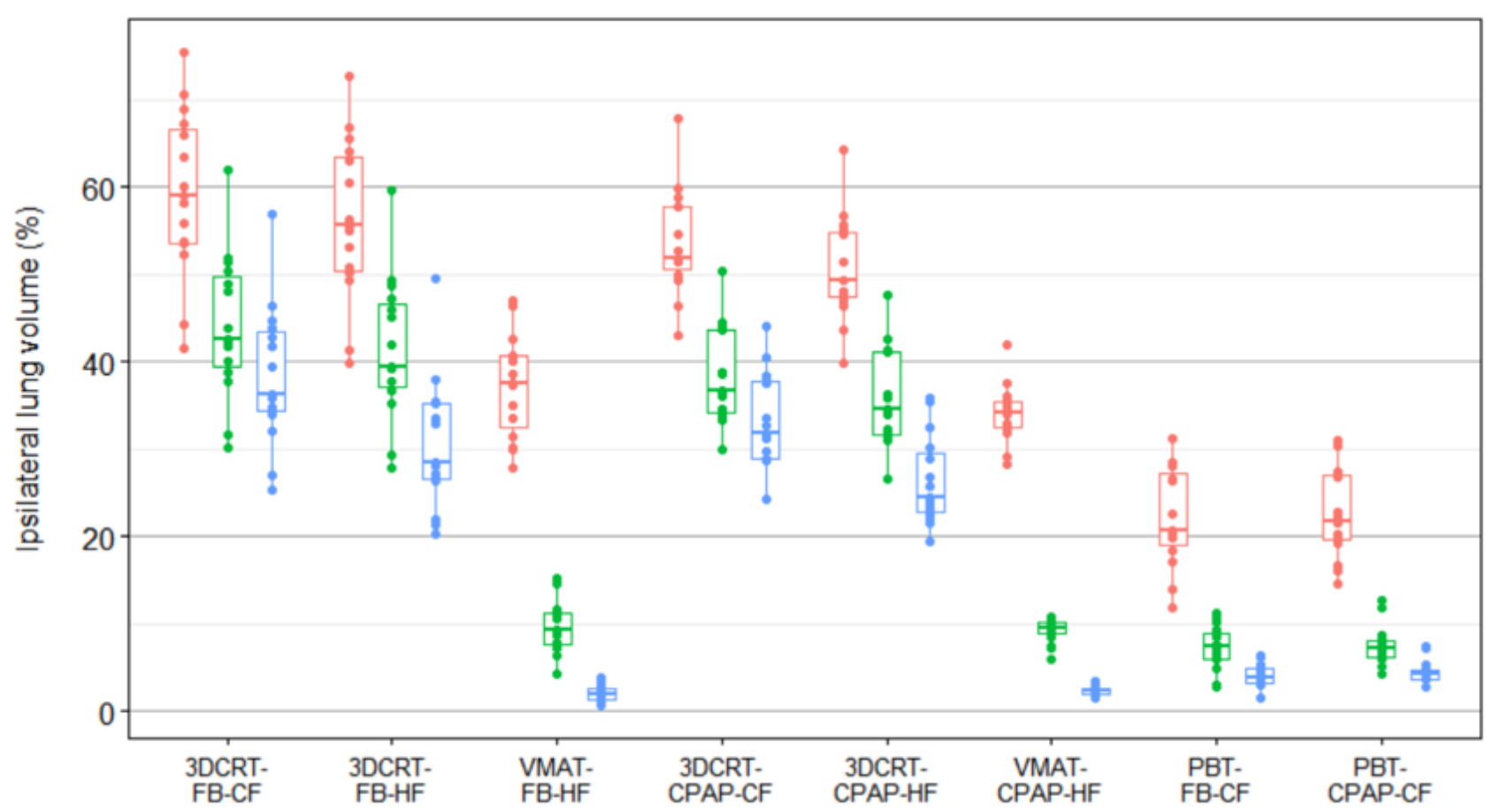

Figure 4

Ipsilateral lung volumes receiving over 5, 20, and 30 Gy per patient per technique

审 V5Gy

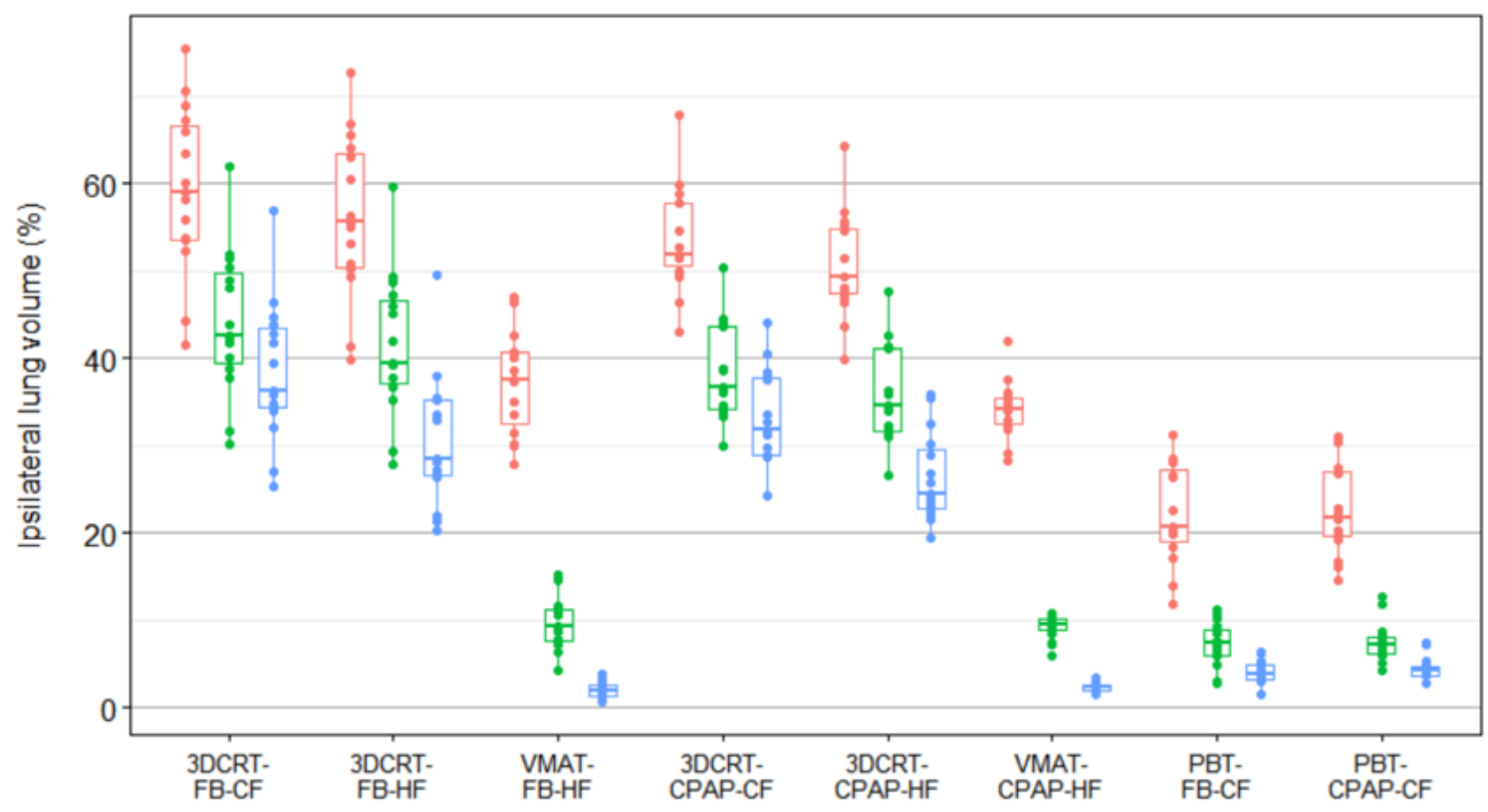

Figure 4 
Ipsilateral lung volumes receiving over 5, 20, and 30 Gy per patient per technique

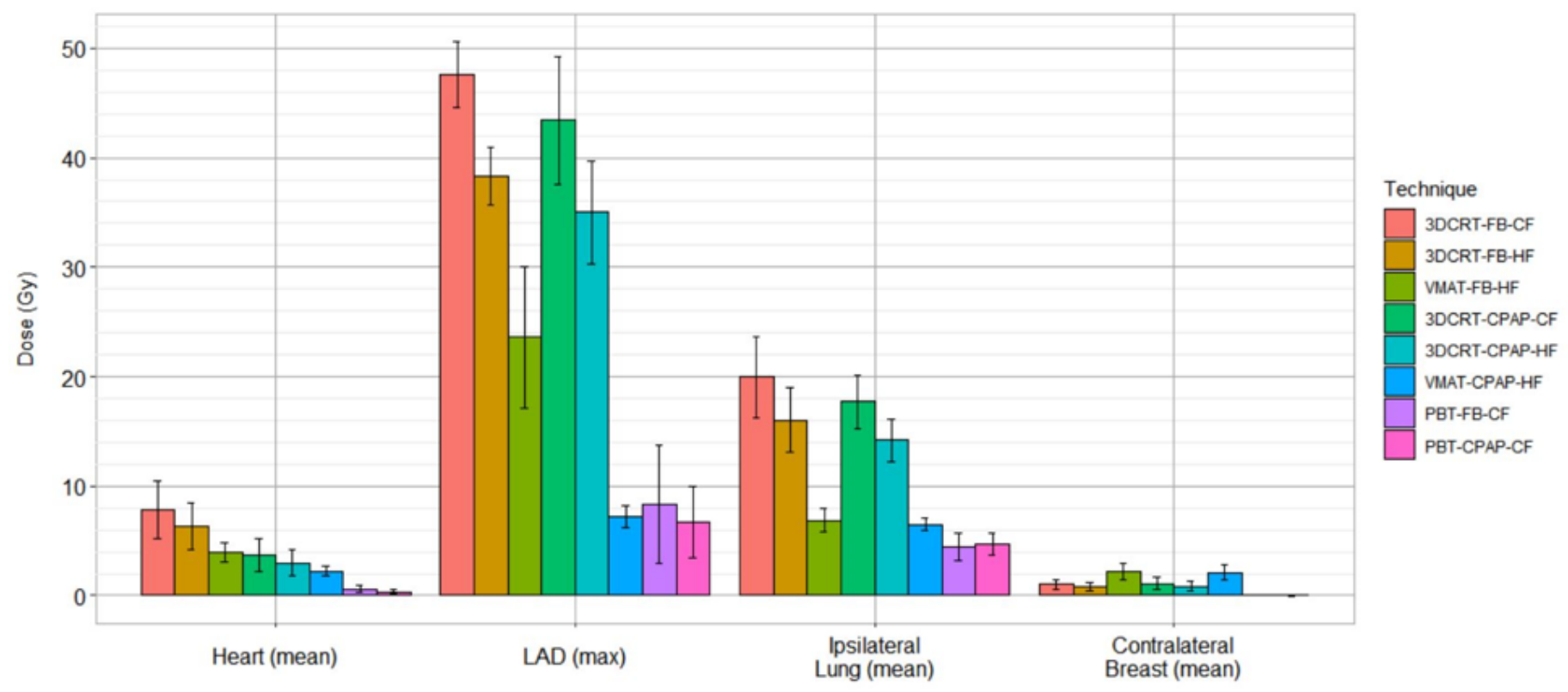

Figure 5

Doses to organs-at-risk per technique, aimed at decreasing the mean heart dose

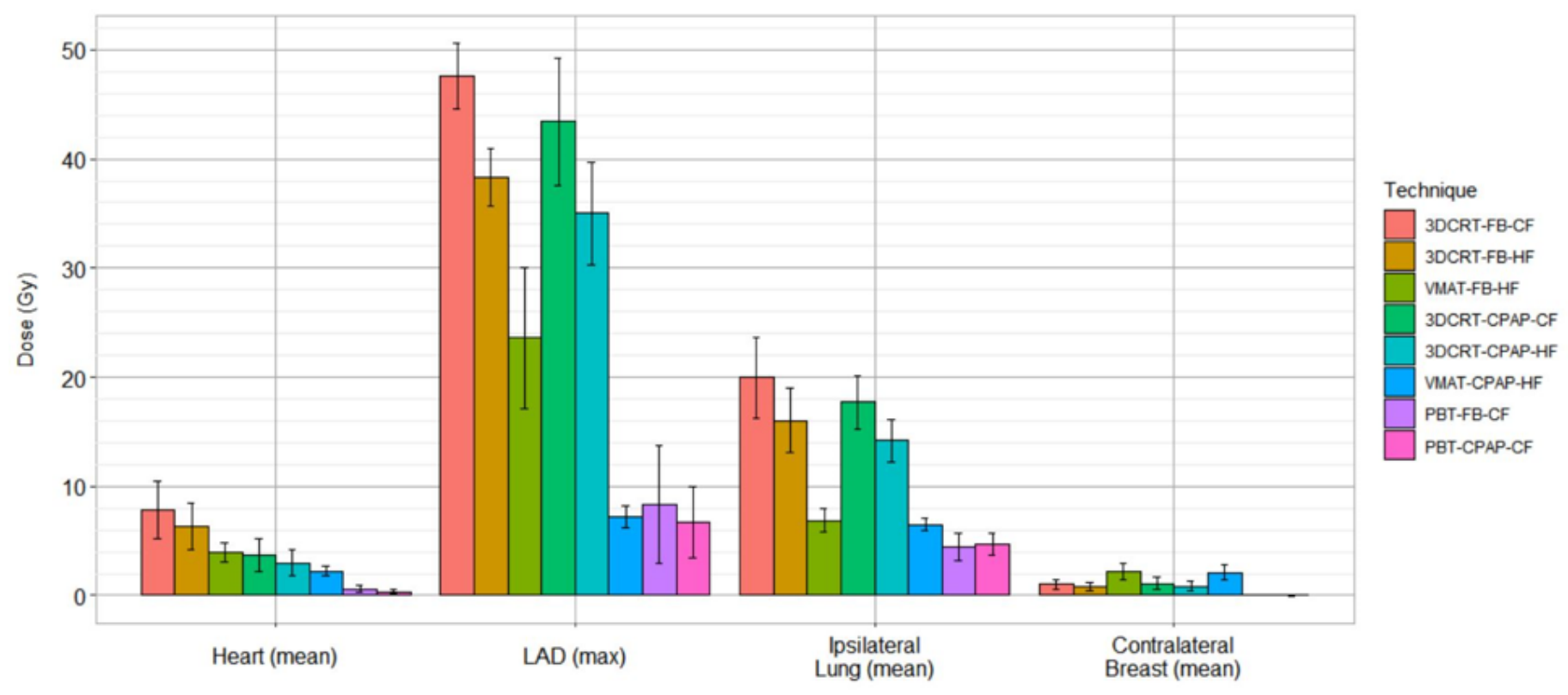

Figure 5

Doses to organs-at-risk per technique, aimed at decreasing the mean heart dose

\section{Supplementary Files}

This is a list of supplementary files associated with this preprint. Click to download. 
- Additionalfile1.docx

- Additionalfile1.docx 